\title{
Association of Apolipoprotein A1 Gene Polymorphism and Coronary Heart Diseases in Patients with Type 2 Diabetes Mellitus \\ Nearmeen M. Rashad ${ }^{1}$, Gehan A. Ebrahem, Amal S. El-Shal'², Mohamed R. H. Abo- Zenar', Islam G. Ahmed ${ }^{3}$, Mohamed G. Hamed ${ }^{1}$ \\ Departments of ${ }^{1}$ Internal Medicine, ${ }^{2}$ Medical Biochemistry and ${ }^{3}$ Cardiology, Faculty of Medicine, Zagazig University, Zagazig, Egypt. \\ * Corresponding author: Mohamed G. Hamed, Mobile: +20 1228588047, E-mail: Mohamed1gaber@gmail.com
}

\begin{abstract}
Background: Type 2 diabetes mellitus (T2DM) is one of the world's most common diseases with a high prevalence and incidence.

Objective: To investigate the association of APOA1 polymorphism (-75 G/A) with dyslipidemia and susceptibility to CAD among Egyptian patients with T2DM.

Patients and Methods: A total number of 72 subjects with T2DM and 72 age and sex matched healthy controls were enrolled for the study. T2DM patients were subdivided to T2DM without CAD $(n=36)$ and T2DM with CAD ( $n=36)$. Genotyping of the APOA1 was performed by polymerase chain reaction-restriction fragment length polymorphism (PCR-RFLP) combined with gel electrophoresis, and then confirmed by direct sequencing.

Results: Our findings revealed that GA and AA genotypes and A allele of APOA1 G-75A were significantly higher in controls than T2DM without CAD cases. Also, GA and AA genotypes and A allele of APOA1 G$75 \mathrm{~A}$ were significantly higher in controls than T2DM with CAD cases. The risk of CAD were significantly lower among patients carrying GA and AA genotypes than those carrying GG genotype (OR (95\% CI): 0.16 (0.06-0.48), $P<0.001$; OR (95\%CI): 0.08 (0.01-0.78), $P<0.01$, respectively). Our findings revealed significantly higher values of HDL in AA genotype of APOA1 G-75A compared to GA and GG. On the other hand, there were significantly lower values of TC and TG in AA genotype of APOA1 G-75A compared to GA and GG.

Conclusions: The individuals with the APOA1 -75 GA and AA genotypes and A allele were likely to have a lower risk of T2DM and CAD as a result of its effect on HDL-C serum levels.
\end{abstract}

Keywords: Apolipoprotein A1, Coronary artery disease, Gene polymorphism, T2DM

\section{INTRODUCTION}

Type 2 diabetes mellitus (T2DM) is considered one of the most common diseases with a high prevalence and incidence worldwide ${ }^{(\mathbf{1})}$. Owing to increased life expectancy, obesity, sedentary life, the incidence of type 2 diabetes is growing at an alarming pace (2). In developing countries, the number of subjects with diabetes is expected to increase by $150 \%$ in the next couples of years. In terms of prevalence of diabetes, Egypt is eighth in the world ${ }^{(3)}$.

The leading cause of death and morbidity worldw ide is coronary artery disease (CAD) (4). Althoughthe exact mechanisms responsible for the onset of CAD are still unclear, it is a

multifactorial disease affected by endogenous and e xogenous factors that interact ${ }^{(5)}$. Age, stress, hypertension, diabetes mellitus, hyperlipidemia, smoking, high alcohol intake, $\mathrm{CAD}$, family history and obesity are the main risk factors for CAD ${ }^{(6)}$.

Lipid metabolism disorder and genetic pred isposition are important risk factors for CAD ${ }^{(7)}$. Genetic risk factors have been estimated to explain approximately $20 \%-60 \%$ of CAD cases ${ }^{(8)}$. Some genes have been investigated for their participation in the development of $\mathrm{CAD}^{\left({ }^{(9)}\right.}$.
The main apolipoprotein portion of high density lipoprotein (HDL) is apolipoprotein A1 (apoA1) and is involved in the transport of reverse cholesterol ${ }^{(10)}$. In the APOA1 gene located on the long arm of chromosome 11, several single nucleotide polymorphisms (SNPs) have so far been identified ${ }^{(\mathbf{1 1})}$. A common-to-A transition of 75 base pairs (bp) located upstream from the APOA1 gene transcription start site has been extensively studied ${ }^{(\mathbf{1 2})}$. In the first intron of the APOA1 gene, which includes the transition of Cto- $\mathrm{T}$ at $+83 \mathrm{bp}$, another polymorphism site was found that has also been shown to affect HDL and apoA1 levels ${ }^{(13)}$.

CAD is the most common fatal complication of T2DM, accounting for almost 60 percent of deaths among patients with T2DM. Patients with T2DM are subject to a two-fourfold higher risk of developing CAD than the overall population ${ }^{(14)}$. Thus, identifying the variables that predispose these patients to develop CAD is of fundamental importance in T2DM management. Insulin controls multiple steps in the metabolism of plasma lipoproteins Dyslipidemia, characterized by hypertriglyceride mia and high density lipoprotein (HDL) 
cholesterol, is frequent in T2DM and can increase the risk of CAD ${ }^{(16)}$.

This is the first research to investigate, to the be st of our knowledge, the association of APOA1 $p$ olymorphism (75 G/A) with dyslipidemia and CAD susceptibility among Egyptian patients with T2DM.

\section{SUBJECTS AND METHODS}

This study included 72 patients with T2DM recruited from diabetes and endocrinology outpatient clinic of Internal Medicine Department of Zagazig University Hospitals and 36 healthy lean control subjects, who were matched to cases by gender and ethnic origin.

The diagnosis of diabetes was according ADA 2020 ; fasting plasma glucose levels of $\geq 126$ $\mathrm{mg} / \mathrm{dL}(7.0 \mathrm{mmol} / \mathrm{L})$ or 2 -h postprandial plasma glucose levels of $\geq 200 \mathrm{mg} / \mathrm{dL}(11.1 \mathrm{mmol} / \mathrm{L})^{(\mathbf{1 7})}$. The enrolled diabetic patients were classified into two groups: 36 patients without CAD and $36 \mathrm{CAD}$ cases were defined as those having severe angiostenosis $(>50 \%)$ in at least one major coronary artery determined by angiography based on ACC/AHA criteria ${ }^{(18)}$.

CAD cases were selected randomly from patients admitted to outpatient clinic of Internal Medicine Department of Zagazig University Hospitals and who fulfilled the inclusion criteria. All patients were subjected to thorough history taking and full clinical assessment including blood pressure and anthropometric variables. BMI was calculated as weight in $\mathrm{kg} /$ height in meters ${ }^{2}$. Patients that were using statins or other lipidlowering drugs discontinued this treatment for at least 45 days before they participated in this study. None of the participants had liver, kidney, thyroid, or any active inflammatory diseases, as well as receiving medications that affect glucose metabolism or for weight reduction or participating in a dietary or exercise programs.

\section{Ethical approval:}

The Ethical Committee of Faculty of Medicine, Zagazig University approved our study protocol, and all participants assigned written informed consent.

\section{Blood samples:}

Blood samples were drawn from all subjects after an overnight fasting and divided into 3 portions: $1 \mathrm{ml}$ of whole blood was collected into evacuated tubes containing EDTA, for genomic DNA extraction and HbA1c; $1 \mathrm{ml}$ of whole blood was collected into evacuated tubes containing fluoride for fasting blood glucose. Serum were separated immediately from remaining part of the sample and stored at $-20^{\circ} \mathrm{C}$ until analysis.

\section{Biochemical analysis:}

We determined fasting blood glucose using the glucose oxidase method (Spinreact, Girona, Spain). Total cholesterol and triglycerides were measured by routine enzymatic methods (Spinreact, Girona, Spain). HDL cholesterol was determined after precipitation of the apoBcontaining lipoproteins. LDL cholesterol was calculated using the Friedewald formula ${ }^{(\mathbf{1 9})}$.

\section{DNA Extraction:}

All the work was carried out in the molecular biology laboratory in the Medical Biochemistry Department. All the reagents were highly purified analytical PCR-materials. All the tubes, tips pipettes used for DNA extraction were DNAse, RNAse free tubes to avoid contamination; purchased from Gentra (Minneapolis. USA).

Genomic DNA was isolated and purified from blood samples using the QIAamp DSP DNA Blood Mini Kit purchased from Qiagen $\mathrm{GmbH}$ (Hilden, Germany). DNA was extracted according to the method of Miller et al. ${ }^{(20)}$ in which standard DNA isolation from $200 \mu \mathrm{l}$ whole blood in standard 45 minutes protocol, including RNase treatment. Expected yield: 5-15 $\mu \mathrm{g}$ DNA.

\section{Each QIAamp DSP DNA Blood Mini procedure comprises 4 steps:}

1. Lysing the cells in the blood sample.

2. Binding the genomic DNA in the cell lysate to the membrane of a QIAamp Mini spin column.

3. Washing the membrane.

4. Eluting the genomic DNA from the membrane.

\section{DNA quantitation:}

For DNA concentration determination and DNA purity evaluation, in $1 \mathrm{ml}$ of deionized water in a quartz cuvette, $20 \mu \mathrm{L}$ of each extracted DNA sample was added and absorption was measured at 260 and $280 \mathrm{~nm}$ wavelengths using the Milton Roy Spectronic 3000 Array. DNA has a maximum absorbance at $260 \mathrm{~nm}$ because the pyrimidine resonance structures and purine bases are responsible for the absorbance. At $260 \mathrm{~nm}$, an absorption of 1.0 gives a DNA concentration of 50 $\mu \mathrm{g} / \mathrm{mL}$. Because of the presence of tyrosine, phenylalanine and tryptophan, proteins absorb maximally at $280 \mathrm{~nm}$ and absorption at this wavelength is used for protein detection in DNA samples. This is done through the A260/A280 ratio determination. This ratio was commonly considered to be between 1.8 and 1.9 for pure double-stranded DNA ${ }^{(21)}$.

Apo lipoprotein A1 polymorphism genotyping: The Egyptian subjects were genotyped by the multiplex amplification refractory mutation system PCRR for Apo lipoprotein A1 
polymorphism. (Multi-ARMS PCR) as described previously.

The upstream internal primers were $\mathrm{P} 1$ (5'ATGCCGAT GACCTGCAGAATT- $3^{\prime}$ ) and P2 (5'-ATGCCGATGACCTGCAGAATC- $\left.3^{\prime}\right)$, the upstream external primers were P3 (5'CGCGGACAT GGAGGACGTTT- $\left.3^{\prime}\right)$ and P4 (5'CGCGGACATGGAGGACGTTC-3'), the downstream primer was P5 (5'GTTCAGTGATTGTCGCTG GGCA-3'). Two tubes were used to duplicate the amplification reactions: tube $\mathrm{A}$ containing $\mathrm{P} 5, \mathrm{P} 1$ and $\mathrm{P}$ 3, tube $\mathrm{B}$ containing P5, P2 and P4. The PCR amplification profile was as follows: initial denaturation for 4 min at $95^{\circ} \mathrm{C}, 35$ cycles with denaturation for $45 \mathrm{~s}$ at $96{ }^{\circ} \mathrm{C}$, annealing for $45 \mathrm{~s}$ at $66^{\circ} \mathrm{C}$, and extension for $45 \mathrm{~s}$ at $72{ }^{\circ} \mathrm{C}$, followed by a final extension cycle for $5 \mathrm{~min}$ at $72{ }^{\circ} \mathrm{C}$. The fragments were separated on 2 percent agarose gels ${ }^{(22)}$.

\section{Statistical analysis}

Data were verified, coded by the researcher and analyzed using IBM-SPSS Statistics for windows, version 23.0 (Copyright IBM Corp., Armonk, N.Y., USA. 2015). Descriptive statistics: Means, standard deviations, and percentages were calculated. Test of significances: Chi-square test was used to compare the difference in distribution of frequencies among different groups. For continuous variables; independent t-test analysis was carried out to compare the means of dichotomous data. There was no specific calculation of the sample size. A significant pvalue was considered when it is equal or less than 0.05 .

\section{RESULTS}

Among studied subjects, in the control group, $69.6 \%$ were male and $30.4 \%$ were female, their mean age was $45.66 \pm 7.56$ year. In the diabetic group, $64.3 \%$ were male and $35.7 \%$ were female, their mean age was $45.58 \pm 8.3$ year. Control and T2DM groups were matched for age, ethnicity and gender.

Among T2DM groups, T2DM without CAD $67.8 \%$ were male and $32.2 \%$ female, their mean age was $47.13 \pm 8.08$ year. In T2DM with CAD, $71.4 \%$ were male and $28.6 \%$ were female, their mean age was $44.02 \pm 8.33$ year. Both diabetic groups were matched for age and gender.

Diabetic patients had significantly higher values of BMI, waist/hip ratio, systolic and diastolic blood pressure as well as lipid profile; TG, TC and LDL compared to control group.

Furthermore, diabetic patients had significantly higher values of FPG, HbA1c and $2 \mathrm{hr}$ PP plasma glucose compared to control group. On the other hand, T2DM patients had significantly lower values of HDL compared to control group (Table 1)

Table (1): Clinical, anthropometric and laboratory characteristics of studied groups

\begin{tabular}{|l|l|l|c|}
\hline \multicolumn{1}{|c|}{ Variables } & \multicolumn{1}{|c|}{$\begin{array}{c}\text { Control group } \\
(\text { Mean } \pm \text { SD) }\end{array}$} & $\begin{array}{c}\text { T2DM group } \\
(\text { Mean } \pm \text { SD) }\end{array}$ & \multicolumn{1}{c|}{ P-value } \\
\hline Systolic blood pressure $(\mathrm{mmHg})$ & $118.33 \pm 6.97$ & $130.56 \pm 18.76$ & $<0.001^{*}$ \\
\hline Diastolic blood pressure $(\mathrm{mmHg})$ & $78.33 \pm 6.97$ & $90.83 \pm 17.82$ & $<0.001^{*}$ \\
\hline Body mass index $\left(\mathrm{kg} / \mathrm{m}^{2}\right)$ & $20.83 \pm 1.15$ & $23.71 \pm 1.91$ & $<0.001^{*}$ \\
\hline Waist circumference & $69.50 \pm 7.06$ & $83.74 \pm 8.74$ & $<0.001^{*}$ \\
\hline Fasting plasma glucose $(\mathrm{mg} / \mathrm{dl})$ & $92.11 \pm 10.46$ & $191.68 \pm 36.35$ & $<0.001^{*}$ \\
\hline 2hr PP plasma glucose $(\mathrm{mg} / \mathrm{dl})$ & $122.97 \pm 16.34$ & $311.07 \pm 9.26$ & $<0.001^{*}$ \\
\hline HbA1c $(\%)$ & $4.60 \pm 0.45$ & $9.77 \pm 2.09$ & $<0.001^{*}$ \\
\hline HDL cholesterol $(\mathrm{mg} / \mathrm{dl})$ & $55.89 \pm 7.28$ & $34.83 \pm 5.91$ & $<0.001^{*}$ \\
\hline LDL cholesterol $(\mathrm{mg} / \mathrm{dl})$ & $85.03 \pm 8.23$ & $120.29 \pm 9.73$ & $<0.001^{*}$ \\
\hline Total cholesterol $(\mathrm{mg} / \mathrm{dl})$ & $156.33 \pm 23.24$ & $251.96 \pm 36.15$ & $<0.001^{*}$ \\
\hline Triglycerides $(\mathrm{mg} / \mathrm{dl})$ & $113.89 \pm 24.76$ & $208.40 \pm 35.21$ & $<0.001^{*}$ \\
\hline
\end{tabular}

T2DM, type 2 diabetes mellitus; $* P<0.05$.

\section{Anthropometric and biochemical characteristics of the T2DM groups}

Among T2DM patients ( $\mathrm{n}=72), 36$ patients had CAD confirmed by coronary angiography, they had significantly longer duration of DM and higher values of systolic as well as diastolic blood pressure compared to T2DM patients without CAD. Furthermore, diabetic patients had significantly lower values of waist circumference and HDL compared to control group (Table 2). 
Table (2): Clinical, anthropometric and laboratory characteristics of T2DM groups

\begin{tabular}{|l|c|c|c|}
\hline & $\begin{array}{c}\text { Without CAD } \\
(\text { Mean } \pm \text { SD })\end{array}$ & $\begin{array}{c}\text { With CAD } \\
(\text { Mean } \pm \text { SD })\end{array}$ & P-value \\
\hline Duration of DM (years) & $5.18 \pm 3.44$ & $10.72 \pm 4.48$ & $<0.001^{*}$ \\
\hline Systolic blood pressure $(\mathrm{mmHg})$ & $119.72 \pm 15.58$ & $141.39 \pm 15.15$ & $<0.001^{*}$ \\
\hline Diastolic blood pressure $(\mathrm{mmHg})$ & $80.56 \pm 14.13$ & $101.11 \pm 15.08$ & $<0.001^{*}$ \\
\hline Body mass index $\left(\mathrm{kg} / \mathrm{m}^{2}\right)$ & $23.76 \pm 1.92$ & $23.66 \pm 1.92$ & 0.826 \\
\hline Waist circumference(cm) & $86.19 \pm 8.08$ & $81.28 \pm 8.79$ & $<0.05^{*}$ \\
\hline Fasting plasma glucose $(\mathrm{mg} / \mathrm{dl})$ & $195.17 \pm 38.54$ & $188.19 \pm 34.20$ & 0.420 \\
\hline 2hr PP plasma glucose $(\mathrm{mg} / \mathrm{dl})$ & $317.06 \pm 8.56$ & $305.08 \pm 78.62$ & 0.525 \\
\hline HbA1c $(\%)$ & $10.17 \pm 2.26$ & $9.37 \pm 1.85$ & 0.106 \\
\hline HDL cholesterol $(\mathrm{mg} / \mathrm{dl})$ & $39.83 \pm 3.07$ & $29.83 \pm 3.16$ & $<0.001^{*}$ \\
\hline LDL cholesterol $(\mathrm{mg} / \mathrm{dl})$ & $123.11 \pm 31.40$ & $117.47 \pm 24.36$ & 0.425 \\
\hline Total cholesterol $(\mathrm{mg} / \mathrm{dl})$ & $250.56 \pm 35.13$ & $253.36 \pm 37.58$ & 0.744 \\
\hline Triglycerides $(\mathrm{mg} / \mathrm{dl})$ & $208.92 \pm 36.22$ & $207.89 \pm 34.67$ & 0.902 \\
\hline
\end{tabular}

T2DM, type 2 diabetes mellitus; CAD coronary artery disease. ${ }^{*} P<0.05$.

Distribution of genotype and allele frequencies of APOA1 (G-75A) in controls and T2DM without CAD cases

In T2DM cases without CAD and healthy controls, genotype and allelic frequencies of APOA1 (G75A) gene polymorphisms are shown in table 3 . In each studied group, the distributions of genotypes were in Hardy-Weinberg equilibrium. Our findings revealed that GA and AA genotypes and A allele of APOA1 G$75 \mathrm{~A}$ were significantly higher in controls than T2DM without CAD cases. The risk of T2DM were significantly lower among patients carrying GA and AA genotypes than those carrying GG genotype or at least carrying one A allele than among patients carrying the $\mathrm{G}$ allele (Table 3 ).

Table (3): Distribution of APOA1 (G-75A) genotypes and allele frequencies in healthy controls and T2DM without CAD

\begin{tabular}{|l|l|c|c|c|c|c|c|}
\hline \multicolumn{2}{|c|}{} & \multicolumn{2}{c|}{ Controls } & \multicolumn{2}{c|}{ T2DM without CAD } & OR (95\% CI) & P \\
\hline \multirow{4}{*}{ APOA1 G-75A } & GG & 33 & $(30.5)$ & 63 & $(58.3)$ & ------ & \\
\cline { 2 - 9 } & GA & 60 & $(55.6)$ & 52 & $(38.9)$ & $0.37(0.13-1.00)$ & $\mathbf{0 . 0 4 3}^{*}$ \\
\cline { 2 - 9 } & AA & 15 & $(13.9)$ & 3 & $(2.8)$ & $0.11(0.01-1.01)$ & $\mathbf{0 . 0 2 2}^{*}$ \\
\cline { 2 - 8 } & G allele & 126 & $(58.3)$ & 168 & $(77.8)$ & & \\
\cline { 2 - 8 } & A allele & 90 & $(41.7)$ & 48 & $(22.2)$ & $0.4(0.19-0.83)$ & $\mathbf{0 . 0 1 1}^{*}$ \\
\hline
\end{tabular}

$* P<0.05$.

Distribution of genotype and allele frequencies of APOA1 (G-75A) in controls and T2DM with CAD cases

Genotype and allelic frequencies of APOA1 (G-75A) genes polymorphisms in T2DM cases with CAD and healthy controls. The genotypes distributions were in Hardy-Weinberg equilibrium in each studied group.

Our findings revealed that GA and AA genotypes and A allele of APOA1 G-75A were significantly higher in controls than T2DM with CAD cases. The risk of CAD were significantly lower among patients carrying GA and AA genotypes than those carrying GG genotype or at least carrying one A allele than among patients carrying the $\mathrm{G}$ (Table 4).

Table (4): Distribution of APOA1 (G-75A) genotypes and allele frequencies in healthy controls and T2DM with CAD.

\begin{tabular}{|l|l|c|c|c|c|c|c|}
\hline \multicolumn{2}{|c|}{} & \multicolumn{2}{|c|}{ Controls } & \multicolumn{2}{c|}{ T2DM with CAD } & OR (95\% CI) & P \\
\hline \multirow{4}{*}{ APOA1 G-75A } & GG & 33 & $(30.5)$ & 81 & $(75)$ & & \\
\cline { 2 - 9 } & GA & 60 & $(55.6)$ & 24 & $(22.2)$ & $0.16(0.06-0.48)$ & $<\mathbf{0 . 0 0 1 *}$ \\
\cline { 2 - 9 } & AA & 15 & $(13.9)$ & 3 & $(2.8)$ & $0.08(0.01-0.78)$ & $<0.01 *$ \\
\cline { 2 - 9 } & G allele & 126 & $(58.3)$ & 186 & $(86.1)$ & & \\
\cline { 2 - 8 } & A allele & 90 & $(41.7)$ & 30 & $(13.9)$ & $0.23(0.10-0.51)$ & $<\mathbf{0 . 0 0 1 *}$ \\
\hline
\end{tabular}

$* P<0.05$. 


\section{DISCUSSION}

T2DM, a leading independent risk factor for cardiovascular disease, is the most frequent cause of morbidity and mortality among diabetic patients (CVD) ${ }^{(23)}$. Egypt's healthcare services are heavily burdened by the growing prevalence of non-communicable diseases (NCDs), including diabetes and cardiovascular diseases, and nearly 41 percent of all deaths in Egypt are attributed to NCDs ${ }^{(24)}$. Modifiable factors have been reported as risk factors for both T2DM and CVDM, such as dyslipidemia, obesity, oxidative stress, smoking, exercise and alcohol consumption, as well as nonmodifiable factors: age, sex, positive family history and genetic predisposition of the person (25). Lipoprotein-related mechanisms have been associated with damage to the cardiovascular system in patients with diabetes ${ }^{(26)}$.

Lipid metabolism disorders, such as elevated TC serum levels ${ }^{(27)}$, TGs ${ }^{(28)}$ LDL-c, ${ }^{(29)}$ and (Apo) $\mathrm{B}^{\left({ }^{(3)}\right)}$ Or low levels of HDL-c ${ }^{(31)}$ and ApoAI ${ }^{(32)}$, have been considered to be important risk factors in the development of atherosclerosis CAD ${ }^{(33)}$.

In reality, for early prediction and identification of at-risk patients and for better clinical management, better understanding of the pathogenesis of CVD and T2DM is of great importance. The goal of the current study was therefore to investigate the association of APOA1 polymorphism $(-75 \mathrm{G} / \mathrm{A}$ and $+83 \mathrm{C} / \mathrm{T})$ with dyslipidemia and CAD susceptibility in Egyptian T2DM patients.

We further sub-group our T2DM patients according to the findings of coronary angiography to T2DM without CAD and T2DM with CAD in order to better elucidate the CAD risk factors. The CAD subgroup of T2DM patients had a substantially longer DM period and higher systolic and diastolic blood pressure relative to T2DM patients without CAD.

This research was the first study examining, to the best of our knowledge, the APOA1G-75A genes mutation in both T2DM with or without CAD for early detection of cardiovascular risks. Accordingly, we can use protective measures of CAD. APOA1 G-75A was the most important finding in our research that GA and AA genotypes and A allele of APOA1 G-75A were significantly higher in controls than T2DD.

Thus, the risk of T2DM was significantly lower among patients carrying GA and AA genotypes than those carrying GG genotype.

In a pilot study in a population in northern India, APOA1 polymorphisms (-75 G/A and +83 $\mathrm{C} / \mathrm{T}$ ) may be susceptible to myocardial infarction ${ }^{(34)}$. In addition, a Brazilian elderly cohort showed that polymorphisms of APOA1 (-75 G/A and +83 $\mathrm{C} / \mathrm{T})$ may be risk factors for hypertension and obesity ${ }^{(35)}$.

The protective effect of HDL-c and ApoAI on the risk of CAD is primarily mediated by the promotion of cholesterol efflux from peripheral cells. Moreover, both HDL-c and ApoAI may have antioxidant, anti-thrombotic and anti-inflammatory properties that may have major anti-atherogenic effects ${ }^{(36)}$. The apo AI gene is located in the 11q23 chromosomal region and is part of the multigene apolipoprotein superfamily, which includes genes encoding exchangeable apolipoproteins such as ApoAI, AII, Cs, and E. The protective effect of GA and AA genotypes and the A allele of APOA1 G$75 \mathrm{~A}$ against $\mathrm{CAD}$ production was an important outcome of our research.

Our results showed that in the control group, the GA and AA genotypes and A allele of APOA1 G-75A were significantly higher than T2DM with CAD cases. Therefore, among patients carrying GA and AA genotypes, the risk of T2DM was significantly lower than those carrying the GG genotype.

Elevated HDL-c levels have been associated with the unusual allele $\mathrm{A}$ of the promoter region polymorphism $75 \mathrm{G} / \mathrm{A}$, and this elevated level is expected to be due to its effects on the transcription of the apo AI gene and increase in ApoAI levels.

Similarly, BiHong et al.'s ${ }^{(37)}$ findings suggested that individuals with the APOA1-75 A allele were likely to have a lower risk of CAD due to its effect on higher serum ApoA1 and HDL-C concentrations. To confirm this finding, further studies are required

Yang et al. (38) investigated APOA1 polymorphism in the same alleles and genotypes in accordance with our study and found GA and AA genotypes and A allele to be more distributed in the control group than the diabetic or diabetic cardiac group so that they are protective.

Also, similar results were described in Angotti et al. ${ }^{(39)}$ study. They track the distribution of different genotypes of APOA1 G 75 A polymorphism in diabetic patients with previous myocardial infarction.

\section{CONCLUSION}

Our results revealed that individual carrying GA and AA genotypes and A allele of APOA1 G$75 \mathrm{~A}$ were significantly higher in controls than both T2DM groups (without or with CAD). Thus, the individuals with the APOA1 -75 GA and AA genotypes and A allele were likely to have a lower risk of T2DM and CAD as a result of its effect on HDL-C. Regarding APOA1 C+83T, TT and CT 
genotypes and $\mathrm{T}$ allele of APOA1 $\mathrm{C}+83 \mathrm{~T}$ were significantly higher in T2DM with CAD cases than control.

\section{REFERENCES}

1. King H, Aubert R, Herman W (1998): Global burden of diabetes, 1995-2025: prevalence, numerical estimates, and projections. Diabetes Care, 21: 1414-31.

2. Sauvanet $\mathbf{J}$ (2003): Congress of the International Diabetes Federation (IDF-Paris 2003). Presse Med., 13: 32(39):1864-8.

3. Guariguata L, Whiting D, Hambleton I et al. (2014): Global estimates of diabetes prevalence for 2013 and projections for 2035. Diabetes Res Clin Pract., 103(2): 137-49.

4. Ordovas J, Schaefer E (1988): Coronary artery disease, lipid disorders and genetic polymorphisms. Ann Biol Clin (Paris), 46, 24-29.

5. BiHong L, Keqi C, Shaohong D et al. (2015): Effect of Apolipoprotein A1 genetic polymorphisms on lipid profiles and the risk of coronary artery disease. Diagnostic pathology, 10 (1): 102-6.

6. Li X, Hong L, Luo S et al. (2014): Impact of admission triglyceride for early outcome in diabetic patients with stable coronary artery disease. Lipids Health Dis., 27: 13:73.

7. Gruzdeva O, Uchasova E, Dyleva Y et al. (2014): Multivessel coronary artery disease, free fatty acids, oxidized LDL and its antibody in myocardial infarction. Lipids Health Dis., 13: 111-14.

8. Kraus W (2000): Genetic approaches for the investigation of genes associated with coronary heart disease. Am Heart J., 140: 27-35.

9. Verdoia M, Cassetti E, Schaffer A et al. (2014): Platelet glycoprotein IIIa Leu33Pro gene polymorphism and coronary artery disease: A metaanalysis of cohort studies. Platelets, 26(6): 530-5.

10. Segrest J, Li L, Anantharamaiah G et al. (2000): Structure and function of apolipoprotein A-I and high-density lipoprotein. Curr Opin Lipidol., 11: 105-15.

11. Karathanasis $S$ (1985): Apolipoprotein multigene family: tandem organization of human apolipoprotein AI, CIII, and AIV genes. Proc Natl Acad Sci USA, 82: 6374-8.

12. Saha N, Tay J, Low P et al. (1994): Guanidine to adenine (G/A) substitution in the promoter region of the apolipoprotein AI gene is associated with elevated serum apolipoprotein AI levels in Chinese non-smokers. Genet Epidemiol., 11: 255-64.

13. Dawar R, Gurtoo A, Singh $R$ (2010): Apolipoprotein A1 gene polymorphism (G-75A and $\mathrm{C}+83 \mathrm{~T})$ in patients with myocardial infarction: a pilot study in a north Indian population. Am J Clin Pathol., 134: 249-55.

14. Pasterkamp G (2013): Methods of accelerated atherosclerosis in diabetic patients. Heart, 99: 743-749

15. Leança $C$, Nunes $V$, Panzoldo $N$ et al. (2013) Metabolism of plasma cholesterol and lipoprotein parameters are related to a higher degree of insulin sensitivity in high HDL-C healthy normal weight subjects. Cardiovasc Diabetol., 12: 173-77.

16. Schofield J, Liu Y, Rao-Balakrishna P et al. (2016): Diabetes Dyslipidemia. Diabetes Ther., 7(2): 203-219.

17. American Diabetes Association (2020): Classification and diagnosis of diabetes: Standards of medical care in diabetes. Diabetes Care, 43(1): 14-31.

18. Arnett $D$, Blumenthal $R$, Albert $M$ et al. (2019): ACC/AHA guideline on the primary prevention of cardiovascular disease: A report of the American College of Cardiology/American Heart Association Task Force on Clinical Practice Guidelines. Circulation, 140(11): 596-646.

19. Friedewald W, Levy R, Fredrickson D (1972): Estimation of the concentration of low-density lipoprotein cholesterol in plasma, without use of the preparative ultracentrifuge. Clin Chem., 18: 499502.

20. Miller S, Dykes D, Polesky H (1988): A simple salting out procedure for extracting DNA from human nucleated cells. Nucleic Acids Res., 16(3): 1215.

21. Southern E (1975): Detection of specific sequences among DNA fragments separated by gel electrophoresis. J Mol Biol., 98(3): 503-17.

22. Donohoe M, Zhang X, McGinnis L et al. (1999): Targeted disruption of mouse Yin Yang 1 transcription factor results in peri-implantation lethality. Mol Cell Biol., 19(10): 7237-44.

23. Lorber D (2014): Importance of cardiovascular disease risk management in patients with type 2 diabetes mellitus. Diabetes Metab Syndr Obes., 7: 169-85.

24. Horejsí B, Ceska R (2000): Apolipoproteins and atherosclerosis. Apolipoprotein E and apolipoprotein (a) as candidate genes of premature development of atherosclerosis. Physiol Res., 49(1): 63-9.

25. Jenkins A, Rowley K, Lyons T et al. (2004): Lipoproteins and diabetic microvascular complications. Curr Pharm Des., 10: 3395-418.

26. Ferreira $\mathrm{C}$, Carvalho $\mathrm{M}$, Fernandes A et al. (2010): Sousa MO. Comparative study of apolipoprotein-E polymorphism and plasma lipid levels in dyslipidemic and symptomatic subjects, and their implication in cardio/cerebro-vascular disorders. Neurochem Int., 56(1):177-82.

27. Kim H, Chang S, Choi E et al. (2005): Association between plasma lipids, and apolipoproteins and coronary artery disease: a cross-sectional study in a low-risk Korean population. Int J Cardiol., 101(3): 435-440.

28. Austin M. (1989): Plasma triglyceride as a risk factor for coronary heart disease. The epidemiologic evidence and beyond. Am J Epidemiol., 129: 249- 259.

29. Achari V, Thakur A (2004): Association of major modifiable risk factors among patients with coronary artery disease - a retrospective analysis. J Assoc Physicians India, 52: 103-108.

30. Walldius G, Jungner I, Holme I et al. (2001): High apolipoprotein B, low apolipoprotein A-I, and 
improvement in the prediction of fatal myocardial infarction (AMORIS study): a prospective study. Lancet, 358: 2026-2033.

31. Assmann G, Schulte H (1992): Relation of highdensity lipoprotein cholesterol and triglycerides to incidence of atherosclerotic coronary artery disease (the PROCAM experience): Prospective Cardiovascular Munster study. Am J Cardiol., 70:733-737.

32. Young S (1990): Recent progress in understanding apolipoprotein

B. Circulation, 82: 1574-1594.

33. Forte T, McCall $M$ (1994): The role of Apolipoprotein AIe containing lipoproteins in atherosclerosis. Curr Opin Lipidol., 5, 354-364.

34. Dawar $R$, Gurtoo A, Singh $R$ (2010): Apolipoprotein A1 gene polymorphism (G-75A and $\mathrm{C}+83 \mathrm{~T})$ in patients with myocardial infarction: a pilot study in a north Indian population. Am J Clin Pathol., 134: 249-55.

35. Chen E, Mazzotti D, Furuya T et al. (2009): Apolipoprotein A1 gene polymorphisms as risk factors for hypertension and obesity. Clin Exp Med., 9: 319-25.

36. Willer C, Sanna S, Jackson A et al. (2008): Newly identified loci that influence lipid concentrations and risk of coronary artery disease. Nat Genet., 40: 161-69.

37. BiHong L, KeQi C, ShaoHong D et al. (2015): effect of apolipoprotein A1 genetic polymorphisms on lipid profiles and the risk of coronary artery disease. Diagnostic Pathology, 10: 102-106.

38. Yang J, Park J, Kim S et al. (2010): Association between Apolipoprotein A1 (APOA1) single nucleotide polymorphisms and schizophrenia in a Korean population. Clin Psychopharmacol Neurosci., 8 (1): 45-48.

39. Angotti E, Mele E, Costanzo F et al. (1994): A polymorphism ( $\mathrm{G} \rightarrow \mathrm{A}$ transition) in the -78 position of the apolipoprotein A-I promoter increases transcription efficiency. J Biol Chem., 269: 17371-17374. 